Vasile Ene, ${ }^{*}$ Ovidius University Constanţa, Romania Current address ${ }^{\dagger}$ : Quellenstraße 18, 63571 Gelnhausen, Germany;

e-mail: gabrielaene@hotmail.com

\title{
ON A PROBLEM OF FAURE AND GUÉNARD
}

\begin{abstract}
In [3], Faure and Guénard put the following problem: Characterize the Denjoy*-integrable functions $f:[a, b] \rightarrow \overline{\mathbb{R}}$ that can be approximated by two Baire 1 functions $g_{\epsilon}$ and $h_{\epsilon}, \epsilon>0$, that are $\mathcal{D}^{*}$-integrable. In the present article we show that this class of functions coincides with the class of all $\mathcal{D}^{*}$-integrable functions $f:[a, b] \rightarrow \overline{\mathbb{R}}$.
\end{abstract}

In [3], Faure and Guénard put the following problem, that can be written as follows:

Problem 1. Characterize the Denjoy*-integrable (short $\mathcal{D}^{*}$-integrable) functions $f:[a, b] \rightarrow \overline{\mathbb{R}}$ that can be approximated by two Baire 1 functions $g_{\epsilon}$ and $h_{\epsilon}$ that are $\mathcal{D}^{*}$-integrable, i.e., $\epsilon>0, g_{\epsilon} \leq f \leq h_{\epsilon}, g_{\epsilon}<\infty, h_{\epsilon}>-\infty$ and

$$
\left(\mathcal{D}^{*}\right) \int_{a}^{b}\left(h_{\epsilon}-g_{\epsilon}\right)(t) d t=(\mathcal{L}) \int_{a}^{b}\left(h_{\epsilon}-g_{\epsilon}\right)(t) d t<\epsilon .
$$

In what follows we show that the class of functions from Problem 1 coincides with the class of all $\mathcal{D}^{*}$-integrable functions $f:[a, b] \rightarrow \overline{\mathbb{R}}$.

We shall use the following well known classes of functions: $\mathcal{C}$ (the continuous functions), $\mathcal{B}_{1}$ (the Baire 1 functions), $A C^{*} G$ and $\underline{A C^{*}} G$ (see for example [6] or [2]).

Definition 1. ([6, p. 241] or $[2$, p. 175]). A function $f:[a, b] \rightarrow \mathbb{R}$ is said to be $\mathcal{D}^{*}$-integrable on $[a, b]$ if there exists $F:[a, b] \rightarrow \mathbb{R}$ such that $F \in A C^{*} G \cap \mathcal{C}$ on $[a, b]$ and $F^{\prime}(x)=f(x)$ a.e. on $[a, b]$. Then $\left(\mathcal{D}^{*}\right) \int_{a}^{b} f(t) d t=F(b)-F(a)$.

Key Words: Denjoy*-integral, Perron type integrals, Baire class 1.

Mathematical Reviews subject classification: 26A39

Received by the editors December 10, 1998

*The author died on November 11, 1998 at the age of 41 years.

† At this address you can contact the author's wife Gabriela Ene. 
Remark 1. There exist a lot of integrals of Perron type that are equivalent to the $\mathcal{D}^{*}$-integral. In [2] we gathered many of these definitions and classified them: for example the Perron type integral defined in [4, p. 158] is called $\mathcal{P}_{7,7}$ in [2] (note that this is the same integral as the one used in [3]), and the $\mathcal{P}_{0}$ integral of [6] is called $\mathcal{P}_{3,3}$ in [2]. In what follows we shall need the $\mathcal{P}_{1,1}$-integral and the $\mathcal{P}_{8,8}$-integral of [2].

Definition 2. $\left[2\right.$, p. 31] Let $P \subseteq[a, b], x_{o} \in P$ and $F: P \rightarrow \mathbb{R} . \quad F$ is

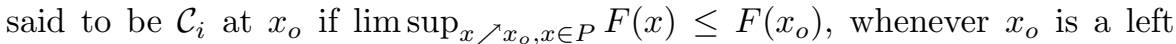
accumulation point for $P$, and $F\left(x_{o}\right) \leq \liminf _{x \backslash x_{o}, x \in P} F(x)$, whenever $x_{o}$ is a right accumulation point for $P$. $F$ is said to be $\mathcal{C}_{i}$ on $P$ if it is so at each point $x \in P$.

Definition 3. [2, pp. 174-175] Let $f:[a, b] \rightarrow \overline{\mathbb{R}}$. We define the following classes of majorants:

- $\overline{\mathcal{M}}_{1}(f)=\left\{M:[a, b] \rightarrow \mathbb{R}: M(a)=0, M \in A C^{*} G \cap \mathcal{C} ; M^{\prime}(x)\right.$ exists (finite or infinite); $\left.f(x) \leq M^{\prime}(x) \neq-\infty\right\}$;

- $\overline{\mathcal{M}}_{8}(f)=\left\{M:[a, b] \rightarrow \mathbb{R}: M(a)=0, M \in \underline{A C^{*}} G \cap \mathcal{C}_{i}\right.$ on $[a, b] ;$ $f(x) \leq M^{\prime}(x)$ a.e. on $\left.[a, b]\right\}$;

We define the following classes of minorants:

- $\underline{\mathcal{M}}_{1}(f)=\left\{m:[a, b] \rightarrow \mathbb{R}:-m \in \bar{M}_{1}(f)\right\} ;$

- $\underline{\mathcal{M}}_{8}(f)=\left\{m:[a, b] \rightarrow \mathbb{R}:-m \in \bar{M}_{8}(f)\right\} ;$

If $\overline{\mathcal{M}}_{1}(f) \neq \emptyset$ (respectively $\overline{\mathcal{M}}_{8}(f) \neq \emptyset$ ), then we denote by $\bar{I}_{1}(b)$ (respectively $\left.\bar{I}_{8}(b)\right)$ the lower bound of all $M(b), M$ in $\overline{\mathcal{M}}_{1}(f)$ (respectively $\overline{\mathcal{M}}_{8}(f)$ ).

If $\underline{\mathcal{M}}_{1}(f) \neq \emptyset$ (respectively $\underline{\mathcal{M}}_{8}(f) \neq \emptyset$ ) then we denote by $\underline{I}_{1}(b)$ (respectively $\underline{I}_{8}(b)$ ) the upper bound of all $m(b), m$ in $\underline{M}_{1}(f)$ (respectively $\underline{M}_{8}(f)$ ).

- $f$ is said to have a $\mathcal{P}_{1,1}$-integral on $[a, b]$ if $\bar{M}_{1}(f) \times \underline{M}_{1}(f) \neq \emptyset$ and $\bar{I}_{1}(b)=\underline{I}_{1}(b)=\left(\mathcal{P}_{1,1}\right) \int_{a}^{b} f(t) d t$.

- $f$ is said to have a $\mathcal{P}_{8,8}$-integral on $[a, b]$ if $\bar{M}_{8}(f) \times \underline{M}_{8}(f) \neq \emptyset$ and $\bar{I}_{8}(b)=\underline{I}_{8}(b)=\left(\mathcal{P}_{8,8}\right) \int_{a}^{b} f(t) d t$.

Remark 2. Following Bruckner [1], let $\Delta=\{f:[a, b] \rightarrow \overline{\mathbb{R}}: f$ is a derivative (finite or infinite), i.e., there exists $F:[a, b] \rightarrow \mathbb{R}$ such that $\left.F^{\prime}=f\right\}$. Then $\Delta \subset \mathcal{B}_{1}$ (see Theorem 2.2.4 of [2, p. 30], or see Corollary 2.4 and Remark 90 of $[1$, p. 90] and the classification of the Zahorski classes, or [7], or [5]). 
Definition 4. We denote by $\underline{\mathcal{B}}_{1}$ the class of all functions $f:[a, b] \rightarrow \overline{\mathbb{R}}$ that are lower semi Baire 1, i.e., the set $\{x \in[a, b]: f(x)>\alpha\}$ is of $F_{\sigma}$-type for $\alpha \in[-\infty,+\infty)$. Similarly $\overline{\mathcal{B}}_{1}$ is the class of all functions $f:[a, b] \rightarrow \overline{\mathbb{R}}$ that are upper semi Baire 1, i.e., the set $\{x \in[a, b]: f(x)<\alpha\}$ is of $F_{\sigma}$-type for $\alpha \in(-\infty,+\infty]$. Clearly $\mathcal{B}_{1}=\underline{\mathcal{B}}_{1} \cap \overline{\mathcal{B}}_{1}$.

Note that in [3], these two classes are denoted by $\mathcal{C}^{-+}$respectively $\mathcal{C}^{+-}$.

Theorem 1. Let $f:[a, b] \rightarrow \overline{\mathbb{R}}$. The following assertions are equivalent:

(i) $f$ is $\mathcal{D}^{*}$-integrable on $[a, b]$;

(ii) For any $\epsilon>0$ there exist two $\mathcal{D}^{*}$-integrable functions $g_{\epsilon}:[a, b] \rightarrow$ $[-\infty,+\infty)$ and $h_{\epsilon}:[a, b] \rightarrow(-\infty,+\infty]$ such that:

- $g_{\epsilon}, h_{\epsilon} \in \Delta$ on $[a, b]$;

- $g_{\epsilon} \leq f \leq h_{\epsilon}$ and $\left(\mathcal{D}^{*}\right) \int_{a}^{b}\left(h_{\epsilon}-g_{\epsilon}\right)(t) d t<\epsilon$;

(iii) Replace in (ii) " $g_{\epsilon}, h_{\epsilon} \in \Delta$ " by " $g_{\epsilon}, h_{\epsilon} \in \mathcal{B}_{1}$ ";

(iv) Replace in (ii) " $g_{\epsilon}, h_{\epsilon} \in \Delta$ " by $\quad g_{\epsilon} \in \overline{\mathcal{B}}_{1}, h_{\epsilon} \in \underline{\mathcal{B}}_{1}$ ".

Proof. (i) $\Rightarrow$ (ii) From [2, Corollary 5.9.1], it follows that $f$ is $\mathcal{D}^{*}$ integrable on $[a, b]$ if and only if $f$ is $\mathcal{P}_{1,1}$ integrable on $[a, b]$, and then

$$
\left(\mathcal{D}^{*}\right) \int_{a}^{b} f(t) d t=\left(\mathcal{P}_{1,1}\right) \int_{a}^{b} f(t) d t
$$

By [2, Lemma 5.7.5], we have that $f$ is $\mathcal{D}^{*}$-integrable on $[a, b]$ if and only if for $\epsilon>0$ there is a pair $\left(M_{\epsilon}, m_{\epsilon}\right) \in \overline{\mathcal{M}}_{1}(f) \times \underline{\mathcal{M}}_{1}(f) \neq \emptyset$ such that $M_{\epsilon}(b)-m_{\epsilon}(b)<$ $\epsilon$. Putting $g_{\epsilon}=m_{\epsilon}^{\prime}$ and $h_{\epsilon}=M_{\epsilon}^{\prime}$ we obtain (ii).

(ii) $\Rightarrow$ (iii) See Remark 2 .

(iii) $\Rightarrow$ (iv) This is evident.

(iv) $\Rightarrow$ (i) Let

$$
M_{\epsilon}(x)=\left(\mathcal{D}^{*}\right) \int_{a}^{x} h_{\epsilon}(t) d t \quad \text { and } \quad m_{\epsilon}(x)=\left(\mathcal{D}^{*}\right) \int_{a}^{x} g_{\epsilon}(t) d t .
$$

Then $\left(M_{\epsilon}, m_{\epsilon}\right) \in \overline{\mathcal{M}}_{8}(f) \times \underline{\mathcal{M}}_{8}(f) \neq \emptyset$. By [2, Lemma 5.7.5] it follows that $f$ is $\mathcal{P}_{8,8}$-integrable on $[a, b]$, and by [2, Corollary 5.9.1], we obtain that $f$ is $\mathcal{D}^{*}$-integrable on $[a, b]$.

Remark 3. Theorem 1, (i), (iv) is in fact Theorem C, 1), 2) of Faure and Guénard [3]. Theorem 1, (i), (iii) solves the Problem 1. 


\section{References}

[1] A. M. Bruckner, Differentiation of real functions, Lect. Notes in Math., vol. 659, Springer-Verlag, 1978.

[2] V. Ene, Real functions - current topics, Lect. Notes in Math., vol. 1603, Springer-Verlag, 1995.

[3] C. A. Faure and F. Guénard, Upper and lower approximation of Perron integrable functions, Real Analysis Exchange 22 (1996/7), no. 2, 626-637.

[4] I. P. Natanson, Theory of functions of a real variable, 2nd. rev. ed., Ungar, New York, 1961.

[5] D. Preiss, Approximate derivatives and Baire classes, Czech. Math. J. 21 (1971), no. 96, 373-382.

[6] S. Saks, Theory of the integral, 2nd. rev. ed., vol. PWN, Monografie Matematyczne, Warsaw, 1937.

[7] Z. Zahorski, Über die Menge der Puncte in welchen die Ableitung unendlich ist, Tôhoku Math. J. 48 (1941), 321-330. 